\title{
Inteligencia emocional y habilidades sociales en adolescentes con alta aceptación social
}

\section{María Alicia Zavala Berbena ${ }^{1}$, María de los Dolores Valadez Sierra $^{2}$, Ma. del Carmen Vargas Vivero ${ }^{1}$}

${ }^{1}$ Facultad de Psicología, Universidad de Guanajuato, León

${ }^{2}$ Departamento de Psicología Aplicada del Centro Universitario de Ciencias de la Salud, Universidad de Guadalajara, Guadalajara

\section{México}




\section{Resumen}

Introducción. La adolescencia es un periodo crítico para el desarrollo individual y social en el que los jóvenes enfrentan diversas situaciones que ponen en juego su Inteligencia Emocional y sus habilidades sociales. Uno de los desafíos más apremiantes del adolescente es la aceptación social que se expresa mediante la popularidad, indiferencia o el rechazo de sus pares. En la aceptación social es importante conocer el papel que juega la Inteligencia Emocional y las Habilidades Sociales, lo que permitirá clarificar las líneas de acción en programas de intervención con adolescentes socialmente vulnerables.

Método. Se aplicó el BarOn EQ-i:YV para valorar la Inteligencia Emocional de un grupo de 62 adolescentes con alta aceptación social (13 años de edad en promedio) que fueron seleccionados mediante nominaciones de pares. Asimismo, fueron evaluados 331 alumnos de grupos naturales, no identificados por su nivel de aceptación social. Se utilizó la escala de Gismero (2002) con el propósito de valorar las habilidades sociales.

Resultados. El análisis estadístico realizado sobre las variables de estudio mostró diferencias significativas en la inteligencia emocional (como medida de auto-informe) en favor del grupo con alta aceptación social con respecto del grupo natural. En el grupo de alta aceptación social se encontraron puntuaciones bajas en las habilidades sociales evaluadas, siendo más baja la habilidad para hacer peticiones. Igualmente, se encontraron correlaciones estadísticamente significativas entre varios de los factores de la prueba de BarOn y los de la Escala de Habilidades Sociales.

Discusión. Las puntuciones elevadas de Inteligencia Emocional en los alumnos del grupo de alta aceptación social son congruentes con los modelos teóricos y los hallazgos encontrados en otros estudios. Se encontraron correlaciones estadísticamente significativas entre los factores de la IE y las Habilidades Sociales que ponen de manifiesto la relación parcial que existe entre estos constructos; sin embargo, las puntuaciones bajas obtenidas por los adolescentes con alta aceptación social en el área de habilidades sociales ponen al descubierto un área potencial para el desarrollo de las habilidades en los mismos, ya que se trata de conductas aprendidas, situacionales y contextualizadas.

Palabras Clave: aceptación social, inteligencia emocional, adolescencia, habilidad social.

Recibido: 26/05/08 Aceptación Provisional: 30/05/08 Aceptación Definitiva: 08/07/08 


\section{Abstract}

Introduction. Adolescence is a critical period for individual and social development in which youngsters face different situations that involve their Emotional Intelligence and social skills. One of the most significant challenges for the adolescent is social acceptance, expressed through popularity, indifference or rejection from peers. In social acceptance it is important to know the role of Emotional Intelligence and social skills, thus helping to clarify lines of action in intervention programs with socially weak adolescents.

Method. Using BArOn EQ-i:YV, emotional intelligence was evaluated for a group of 62 adolescents with high social acceptance (average age 13). These were selected by partner nominations. Similarly, 331 students from natural groups, not identified by their degree of social acceptance, were also evaluated. Gismero's scale (2002) was used to evaluate their social skills.

Results. Statistical analyses of the variables showed significant differences in emotional intelligence (self-report measure) in favor of the higher social acceptance group as compared to the natural group. In the former group, lower scores were found for several social skills, with the request-making skill being lowest. In the same way, statistically significant correlations were found among several factors from BarOn's test and from the social skills scale.

Discussion. The high emotional intelligence scores of students in the higher social acceptance group are congruent with theoretical models and with results from other studies. Significant statistical correlations were found among the EI factors and social skills that showed a partial relationship between these constructs; however, the low scores in the area of social skills observed in adolescents with higher social acceptance uncover an opportunity for developing such skills with them, since these skills represent learned, situational and contextualized behaviors.

Keywords: social acceptance, emotional intelligence, adolescents, social skill.

Received: 05/26/08 Initial Acceptance: 05/30/08 Final Acceptance: 07/08/08 


\section{Introducción}

\section{Aproximación al concepto de Inteligencia Emocional}

La Inteligencia Emocional [IE] es un constructo que en las dos últimas decadas ha destacado por su capacidad amplia para explicar el bienestar personal y el éxito social en las organizaciones empresariales, la familia y la escuela. En el contexto educativo son de particular interés las relaciones positivas establecidas entre la IE y el logro académico, la prevención de conductas de riesgo, el ajuste social y la calidad y cantidad de las relaciones interpersonales (Fernández-Berrocal, Extremera y Palomera, 2008). Uno de los marcos teóricos de referencia más importantes en el estudio de la IE es el Modelo de Habilidades propuesto por Mayer y Salovey (1997), quienes la definieron como un equipo de habilidades cognitivas para percibir, usar, comprender y manejar las emociones, cuyas consecuencias son adaptativas para el sujeto; asimismo, postularon cuatro dimensiones o habilidades básicas que favorecen la función adaptativa: 1) percepción y expresión de las emociones; 2) facilitación emocional del pensamiento; 3) comprensión emocional; y, 4) gestión de las emociones. Posteriormente, asociados con Caruso definieron la IE como la habilidad para identificar, procesar y manejar las emociones propias y las de otros (Mayer, Salovey y Caruso 2000). Este último enfoque acrecentó el concepto de la IE ya que implicó tanto las dimensiones cognitivas para la percepción, comprensión y gestión de la propia información emocional como su utilidad para relacionarse con los demás.

Otro de los acercamientos teóricos, difundido ampliamente en el área de la inteligencia emocional, es el Modelo Mixto en el que destacan autores como Goleman, Petrides y Bar-On. En dichos modelos se congregan, además de habilidades cognitivas, otras variables relacionadas con la personalidad tales como la asertividad, la motivación y la autoestima. Goleman (2000), quien popularizó el término de inteligencia emocional, es uno de los representantes inciales del Modelo Mixto; definió la IE como la habilidad para automotivarse y la tenacidad para encarar frustraciones; controlar impulsos y relegar la gratificación; regular los propios estados de ánimo y controlar el miedo para dejar fluir la capacidad de pensar. Su orientación tuvo gran éxito en el campo las organizaciones. Petrides y Furnham (2001) consideran que la IE es una constelación de emociones, percepciones y rasgos de la personalidad, subrayando entre los últimos la empatía, la felicidad y el optimismo. Para Bar-On (1997) la IE es un conjunto de capacidades emocionales, personales e interpersonales que influyen en la capacidad total del individuo para hacer frente a las demandas y a las presiones del medio ambiente. 
Bar-On propuso un modelo de factores en el que inciden cinco dimensiones de la IE: 1) Habilidades Intrapersonales; 2) Habilidades Interpersonales; 3) Manejo del Estrés; 4) Adaptabilidad; y, 5) Estado de Ánimo General. Este último enfoque se destaca por el énfasis que atribuye a la IE en la adaptación del individuo a diferentes situaciones de naturaleza emocional y social.

El Modelo de Habilidades y el Modelo Mixto han arrojado luz sobre el concepto de inteligencia emocional, ambos tratan de explicar los factores que determinan el ajuste social exitoso y sus desarrollos nos permiten una comprensión de la complejidad de su objeto de estudio. De acuerdo con Fernández-Berrocal y Extremera (2006) la existencia de concepciones teóricas dispares sobre el concepto de IE debe ser considerada como una muestra de madurez de un campo de estudio incipiente que está en pleno desarrollo; sin embargo, queda pendiente una reflexión sobre si se trata únicamente de una cuestión de énfasis en la cognición, la personalidad o ambas, o si sus diferencias conceptuales son tan profundas que merecen diferente denominación.

\section{Inteligencia emocional y adolescencia}

La IE ha sido ampliamente estudiada con adultos en el ámbito de las organizaciones; sin embargo, todavía son pocos los estudios que se han realizado con adolescentes, entre otras razones, porque los instrumentos de que se dispone para acceder al estudio en este nivel de edad son adaptaciones de instrumentos para adultos; o bien, son muy recientes. Entre los estudios de la Inteligencia Emocional en adolescentes se destaca el de Harrod y Scheer (2005) quienes valoraron la IE en 200 adolescentes para relacionarlo con características demográficas. Estos autores encontraron relaciones positivas de la IE con el nivel educativo de los padres y los ingresos del jefe de familia. Por otra parte, Martinez-Pons (1998) analizó los componentes de la IE incluyendo dos aspectos: la orientación a sí mismo y la orientación hacia los otros. Concluyó que la orientación hacia los otros es mejor predictor de la calidad de las relaciones interpersonales de los adolescentes, mientras que la orientación a sí mismo correlacionó con orientación a la tarea y reflejó una preocupación por el dominio y la mejora personal.

Uno de los temas más recurrentes del estudio de la IE en la adolescencia es el de su relación con la vida escolar. Parker et al. (2004) encontraron una fuerte asociación entre el éxi- 
to académico de los adolescentes con las principales dimensiones de la IE. Los alumnos quienes obtuvieron mayor promedio académico obtuvieron puntuaciones elevadas de IE en las áreas: interpersonal, adaptabilidad y manejo del estrés. Asimismo, Petrides, Frederickson y Furnham (2004) estudiaron el rol de la IE en el rendimiento académico y las conductas escolares disruptivas. Se encontraron relaciones moderadas entre IE y las habilidades cognitivas y rendimiento académico; además, los jóvenes con mayor puntaje en IE fueron menos propensos a presentar faltas injustificadas o a ser expulsados de la escuela.

\section{Inteligencia emocional y habilidades sociales}

Las habilidades sociales son conductas observables, aprendidas y utilizadas en los intercambios sociales para obtener fines concretos. Una habilidad es "una rutina cognitiva o conductual concreta que forma parte de una estrategia más amplia” (Trianes, de la Morena y Muñoz, 1999, p. 18). Para el desempeño social competente es necesario poner en juego patrones de respuesta específicos tales como: la habilidad para defender los propios derechos, la habilidad para hacer peticiones, la habilidad para decir no y cortar interacciones, entre otras. Se trata de expresiones observables de la inteligencia social. Thorndike (1920, p. 228) definió la inteligencia social como "la habilidad para comprender y dirigir hombres y mujeres, niños y niñas, y actuar pruedentemente en las relaciones humanas". La concepción de la inteligencia social de Thorndike ha fundamentado varios estudios en el área y en esencia es una de las más utilizadas; sin embargo, actualmente, se considera que la inteligencia social no consiste en una habilidad única. Weis y Süß (2005) con base en la revisión de la literatura y en el análisis de trabajos empíricos propusieron un modelo de inteligencia social que contiene cinco dimensiones cognitivas: comprensión social, memoria social, percepción social, creatividad social (o flexibilidad), y conocimiento social, cuya presencia e interacción determina el comportamiento social; igualmente, reconocieron la presencia de factores adicionales tales como el contexto social, la personalidad, los intereses, las metas y la experiencia de los individuos, mismos que regulan las fuerzas del comportamiento social.

En cuanto a la relación entre IE y habilidades sociales, Davies, Stankov y Roberts (1998) realizaron un estudio utilizando el IPT-15 y una serie de tareas de percepción emocional facial. Los autores encontraron una ausencia de correlación entre ambas pruebas $\mathrm{r}=-.09$. Sin embargo, en el trabajo de Weis y Süß (2005) se descubrieron correlaciones significativas entre los componentes de la inteligencia social (memoria social y comprensión social) y la 
inteligencia emocional; por otra parte, Charbonneau y Nicol (2002) encontraron que la IE está asociada con liderazgo potencial; además, Mestre, Guil, Lopes y Salovey (2006) encontraron que las habilidades para comprender y manejar emociones (medidas por el MSCEIT) se relacionaron positivamente entre las mujeres con la denominación como amiga por parte de los compañeros. Todavía existe poca investigación que analice la relación entre estas variables y los resultados son inconsistentes, mismos que se atribuyen tanto a las diferencias conceptuales como a las estrategias de medición.

\section{Aceptación social en la adolescencia}

La adolescencia es un período crítico para la manifestación y el desarrollo de la inteligencia emocional y de las habilidades sociales. Por un lado, los adolescentes han dejado atrás las formas de socialización generalizada de la niñez para relacionarse con sus pares en la búsqueda de comprensión y aceptación social; el entendimiento de lo social se vuelve mucho más complejo, ya que en la adolescencia se abandona el acatamiento pasivo de las normas sociales en favor de un comportamiento más crítico y desafiante de las normas que enfrentan; por otra parte, tienen mayor presión por parte de padres y profesores ya que se espera de ellos comportamientos más elaborados. Entre los problemas más comunes en esta etapa se destacan la ausencia de asertividad, las dificultades en la solución de problemas y la ansiedad para relacionarse con compañeros o compañeras del sexo opuesto (Cristoff et al., 1985).

La IE y las habilidades sociales juegan un papel importante en la capacidad individual para encarar los desafíos de la edad adolescente ya que aportan beneficios importantes como es el caso de la aceptación social. La aceptación social es la condición personal de un individuo con respecto de un grupo de referencia; se relaciona con el liderazgo, la popularidad, el compañerismo, la jovialidad y el respeto. De acuerdo con Zirkel (2000) las personas con alta aceptación social son supuestamente poseedoras de basto conocimiento acerca de ellos mismos y del mundo social en el que viven y utilizan activamente este conocimiento para dirigir su conducta hacia el logro de metas deseadas.

La aceptación social entre los iguales es un reflejo de un estatus psicométrico producto de las valoraciones sociales, formulado especialmente por los compañeros de grupo frente a diferentes situaciones y contextos. Es un constructo bipolar con distintas intensidades que van desde la popularidad hasta el rechazo social. Puede ser expresado mediante un índice numéri- 
co que indica el grado de aceptación que tiene un individuo frente a sus pares. El nivel más alto es el del alumno aceptado o popular, quien recibe el mayor número de valoraciones o nominaciones positivas por parte de sus compañeros. Ésta se relaciona con mostrar comportamientos de ayuda, ser considerado con los compañeros, seguir las reglas de los juegos e implicarse activamente en la interacción positiva con los iguales (Trianes, de la Moreña y Muñoz, 1999). Es fundamental conocer el papel que juega la inteligencia emocional en adolescentes con alta aceptación social ya que permite clarificar sus relaciones y trazar las líneas de acción que se traducirán en programas de intervención con adolescentes, especialmente útiles en poblaciones socialmente vulnerables.

A partir de lo anterior se formuló la siguiente hipótesis central que se someterá a constrastación en el presente estudio: existen diferencias significativas en las puntuaciones de la inteligencia emocional en favor del grupo de alumnos con alta aceptación social comparado con sus pares de grupos naturales no identificados por su nivel de aceptación social.

Asimismo, se planteraron dos objetivos: el primer objetivo fue valorar el nivel de inteligencia emocional en alumnos con alta aceptación social en comparación con alumnos de grupos naturales; el segundo objetivo consitió en valorar la relación entre inteligencia emocional y habilidades sociales en el grupo de alumnos con alta aceptación social. Este último objetivo fue planteado debido a los resultados contradictorios reportados en las investigaciones del área, de donde se desprende la necesidad de realizar estudios más minuciosos en los que las medidas utilizadas se correspondan claramente con las formas de conceptualizar ambos constructos.

\section{Método}

\section{Participantes}

La muestra de estudio estuvo constituida por 62 adolescentes del primer grado de enseñanza secundaria, inscritos en escuelas públicas de contexto urbano (39 mujeres y 23 hombres). El rango de edad de estos alumnos era de 13 a 16 años, con una media de 13.3 años de edad $(\mathrm{DE}=0.70)$. Se eligió a estos adolescentes de un universo de 2670 alumnos distribuidos en 62 grupos naturales. Los alumnos fueron seleccionandos mediante un cuestionario para 
sociograma, que fue cumplimentado por los propios compañeros. Se eligió a un alumno por cada grupo escolar con un promedio de 43 alumnos por grupo.

El grupo de comparación estuvo formado por 331 alumnos (192 mujeres y 139 hombres) de grupos naturales, no identificados por su grado de aceptación social, incluidos en el universo anterior. El rango de edad de estos alumnos fue de 12 a 15 años, con una media de 13.1 años de edad $(\mathrm{DE}=0.42)$.

\section{Instrumentos}

Para valorar la IE se utilizó el BarOn EQ-iYV que es un instrumento de autoinforme compuesto por 60 ítems que evalúan la IE con cuatro escalas: Intrapersonal, Interpersonal, Manejo del Estrés, Adaptabilidad y otras tres escalas que valoran: Estado de Ánimo, Impresión Positiva e Inconsistencia. El cuestionario cuenta con propiedades psicométricas satisfactorias, sus escalas para el grupo de edad de 13 a 15 años tienen índices de consistencia interna entre .80 y .91 . Su validez se realizó mediante técnicas de análisis factorial y revisando su relación con otras medidas de IE, factores de personalidad e índices de conductas problemáticas. Para su calificación se utilizaron los baremos de una tipificación local, realizada con 331 adolescentes (191 mujeres y 140 hombres).

Para la evaluación de las Habilidades Sociales se utilizó la Escala de Habilidades Sociales de Gismero (2002). Es un instrumento de autopercepción propio para adolescentes y adultos. Está compuesto por 33 ítems que valoran 6 formas de comportamiento socialmente habilidoso: Autoexpresión en Situaciones Sociales, Defensa de los Propios Derechos como Consumidor, Expresión de Enfado o Disconformidad, Decir No y Cortar Interacciones, Hacer Peticiones e Iniciar Interacciones Positivas con el Sexo Opuesto. En la justificación estadística de este instrumento se calcularon coeficientes de confiabilidad con el procedimiento Alfa de Cronbach con valor de .88 para todos los ítems considerados como unidad conceptual. La validez de constructo se realizó mediante descripciones y lista de adjetivos con las que se encontraron correlaciones positivas y estadísticamente significativas. Para su calificación se utilizaron las normas para jóvenes y adolescentes propuestas por Gismero (2002).

\section{Procedimiento}


Para la selección de alumnos con alta aceptación social se realizaron 62 sociogramas grupales. El instrumento que se utilizó para esta técnica fue un cuestionario con los siguientes ítems: Es un compañero/a de tu clase que te gustaría que fuera el jefe o representante del grupo; 2) es un compañero/a de tu clase que tiene más amigos que los demás; 3) es un compañero/a de tu clase que comparte todo lo que tiene; y, 4) es un compañero/a de tu clase que respeta siempre a los alumnos y maestros de la escuela. A partir del sociograma se eligió en cada grupo al alumno con el mayor número de nominaciones, derivado de la suma de los cuatro ítems aplicados. Posteriormente se aplicó el Cuestionario de Inteligencia Emocional (Bar-On y Parker, 2004) y la Escala de Habilidades Sociales (Gismero, 2002) a los 62 alumnos seleccionados. Igualmente, se realizó una aplicación del Bar-On al grupo comparativo de alumnos de grupos naturales, no identificados por su grado de aceptación social.

\section{Resultados}

Los resultados en la valoración de la inteligencia Emocional en el grupo de adolescentes con alta aceptación social se presentan en la Gráfica 1. En general, se observaron puntuaciones altas en las escalas del BarOn EQ-iYV. Se obtuvo un percentil de 80 en la puntuación mediana del Coeficiente Emocional de este grupo, mientras que en los alumnos del grupo natural se obtuvo una puntuación percentilar de 50 . 


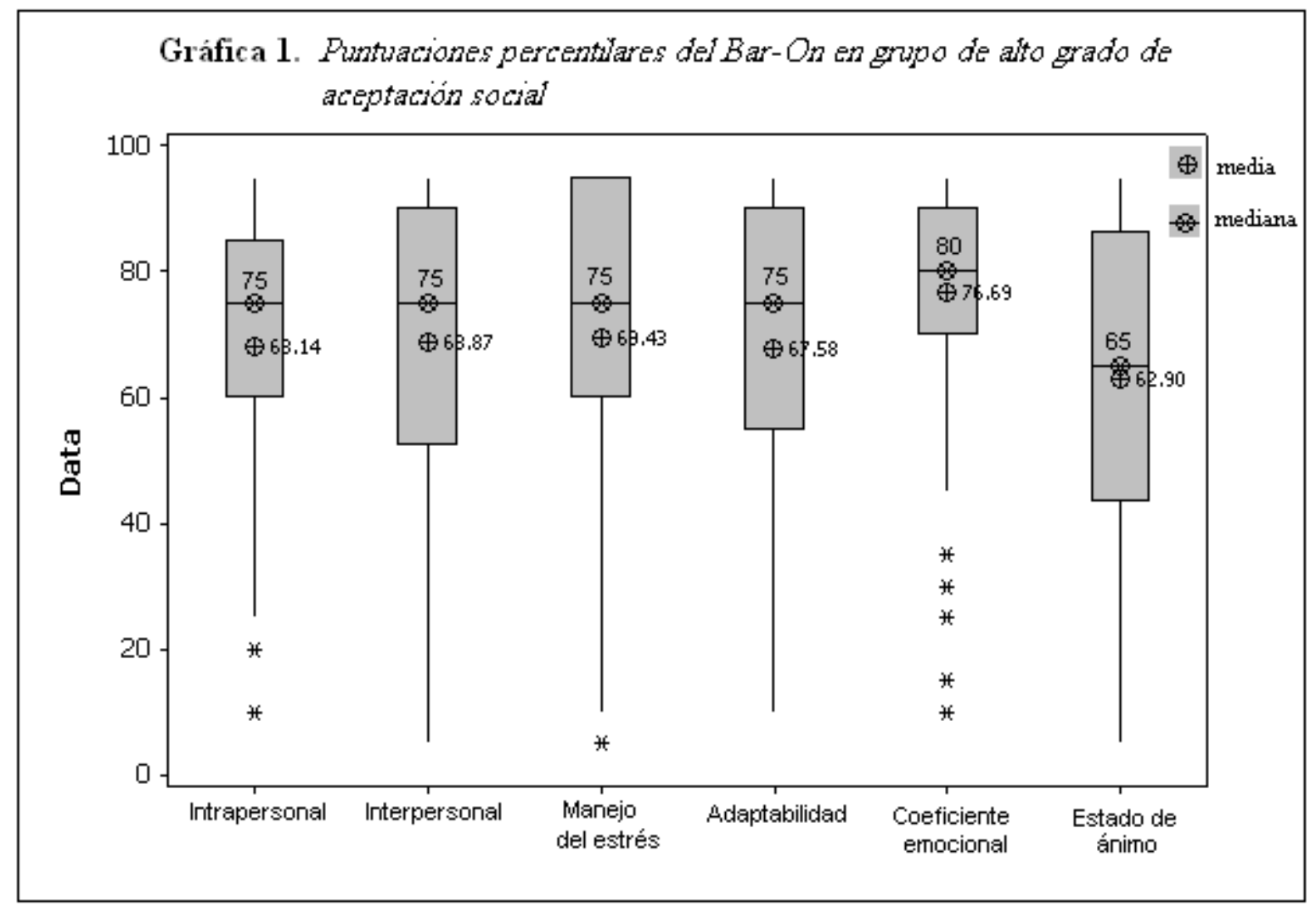

Se calcularon medias y desviaciones estándar para cada uno de los factores del Bar-On y se compararon los puntajes de cada uno de estos por sexo intra e intergrupo y a su vez entre grupos con la prueba de Mann-Whitney. En las comparaciones intragrupo, en el caso de alumnos de alta aceptación social, por sexo, se observaron mayores puntuaciones en los varones en los factores de Adaptabilidad, Coeficiente Emocional y Estado de Ánimo; en el caso de las mujeres se observaron mayores puntuaciones en el factor Interpersonal, Intrapersonal y Manejo del Estrés; sin embargo, ninguna de estas diferencias fue estadísticamente significativa. Para el grupo natural, por sexo, se observaron diferencias estadísticamente significactivas en los factores Intrapersonal $(z=-2.119 ; p=0.034: p<.05)$ e Interpersonal $(z=-2.590 ; p=$ $0.010 ; \mathrm{p}<.01)$ calificando más alto en las mujeres, y en el factor Estado de Ánimo $(\mathrm{z}=-$ $2.675 ; \mathrm{p}=0.007 ; \mathrm{p}<.01)$ calificando más alto los varones.

Se compararon las puntuaciones naturales obtenidas en los diferentes factores del BarOn entre grupos y entre sexos (Ver Tabla 1). Se encontraron diferencias significactivas entre el grupo natural y el grupo de alta aceptación social en todos los factores del Bar-On, calificando más alto estos últimos, lo que confirmó la hipótesis del presente estudio. De igual for- 
ma, se realizaron comparaciones entre sexo y entre grupos, donde para las mujeres se encontraron diferencias significativas en todos los factores del Bar-On, calificando más alto las mujeres de grupo de alta aceptación social, en el caso de los varones se observaron diferencias significativas en los factores Intrapersonal $(\mathrm{z}=-2.649 ; \mathrm{p}=0.008 ; \mathrm{p}<.01)$, Intepersonal $(\mathrm{z}=$ -3.463; $\mathrm{p}=.001 ; \mathrm{p}<.001)$, Manejo del Estrés $(\mathrm{z}=-3.152 ; \mathrm{p}=0.002 ; \mathrm{p}<.01)$, Adaptabilidad $(\mathrm{z}=-3.653 ; \mathrm{p}=0.000 ; \mathrm{p}<.001)$ y Coeficiente emocional $(\mathrm{z}=-4.923 ; \mathrm{p}=0.000 ; \mathrm{p}<.001)$, donde los varones del grupo de alta aceptación social calificaron más alto.

Tabla 1. Puntuaciones naturales obtenidas en los diferentes factores del Bar-On en el grupo natural y en el grupo de alta aceptación social.

\begin{tabular}{|c|c|c|c|c|c|c|c|c|c|c|c|c|}
\hline \multirow{3}{*}{ Factores } & \multicolumn{6}{|c|}{ Grupo Natural } & \multicolumn{6}{|c|}{ Grupo de alta aceptación social } \\
\hline & \multicolumn{2}{|c|}{$\begin{array}{l}\text { Masculino } \\
(n=139)\end{array}$} & \multicolumn{2}{|c|}{$\begin{array}{l}\text { Femenino } \\
(n=192)\end{array}$} & \multicolumn{2}{|c|}{$\begin{array}{c}\text { Total } \\
(n=331)\end{array}$} & \multicolumn{2}{|c|}{$\begin{array}{l}\text { Masculino } \\
\qquad(n=23)\end{array}$} & \multicolumn{2}{|c|}{$\begin{array}{l}\text { Femenino } \\
\qquad(n=39)\end{array}$} & \multicolumn{2}{|c|}{$\begin{array}{l}\text { Total } \\
(n=62)\end{array}$} \\
\hline & $M$ & $\mathrm{DE}$ & $M$ & $\mathrm{DE}$ & $M$ & $\mathrm{DE}$ & $M$ & $\mathrm{DE}$ & $M$ & $\mathrm{DE}$ & M & DE \\
\hline Intrapersonal & 13.28 & 2.67 & 13.99 & 2.87 & 13.69 & 2.80 & $15.09^{* *}$ & 3.37 & $15.46^{*}$ & 2.68 & $15.32^{* * *}$ & 2.94 \\
\hline Interpersonal & 32.82 & 5.25 & 34.43 & 5.80 & 33.75 & 5.62 & $37.13^{* \star *}$ & 5.03 & $38.15^{\star * *}$ & 4.63 & $37.77^{\star \star *}$ & 4.76 \\
\hline $\begin{array}{l}\text { Manejo del } \\
\text { estrés }\end{array}$ & 33.24 & 5.25 & 32.23 & 5.11 & 32.66 & 5.18 & $33.22^{* *}$ & 4.94 & $35.67^{* * *}$ & 4.80 & $36.24^{* * *}$ & 4.87 \\
\hline Adaptabilidad & 26.53 & 5.24 & 26.70 & 5.10 & 26.63 & 5.15 & $31.09^{* * *}$ & 5.05 & $29.44^{* *}$ & 4.96 & $30.05^{* * *}$ & 5.02 \\
\hline $\begin{array}{l}\text { Coeficiente } \\
\text { Emocional }\end{array}$ & 51.90 & 5.91 & 52.79 & 6.28 & 52.41 & 6.13 & $59.04^{* * *}$ & 5.55 & $58.33^{* * *}$ & 5.75 & $58.60^{* * *}$ & 5.64 \\
\hline $\begin{array}{l}\text { Estado de } \\
\text { ánimo }\end{array}$ & 45.69 & 6.69 & 43.53 & 7.39 & 44.44 & 7.17 & 48.30 & 5.88 & $46.95^{* *}$ & 6.58 & $47.45^{\star * *}$ & 6.325 \\
\hline
\end{tabular}

En la Gráfica 2 se presentan las puntuaciones obtenidas en los factores de la Escala de Habilidades Sociales en el grupo de alumnos con alta aceptación social. En la mediana de la puntuación global el percentil fue de 30 puntos con una media de 38. Se destacan bajas puntuaciones en la Habilidad para Hacer Peticiones (Mdn, percentil 25), Expresión de Enfado y Disconformidad (Mdn, percentil 30) y en la Habilidad para Iniciar Interacciones Positivas con el Sexo Opuesto (Mdn, percentil 30). 


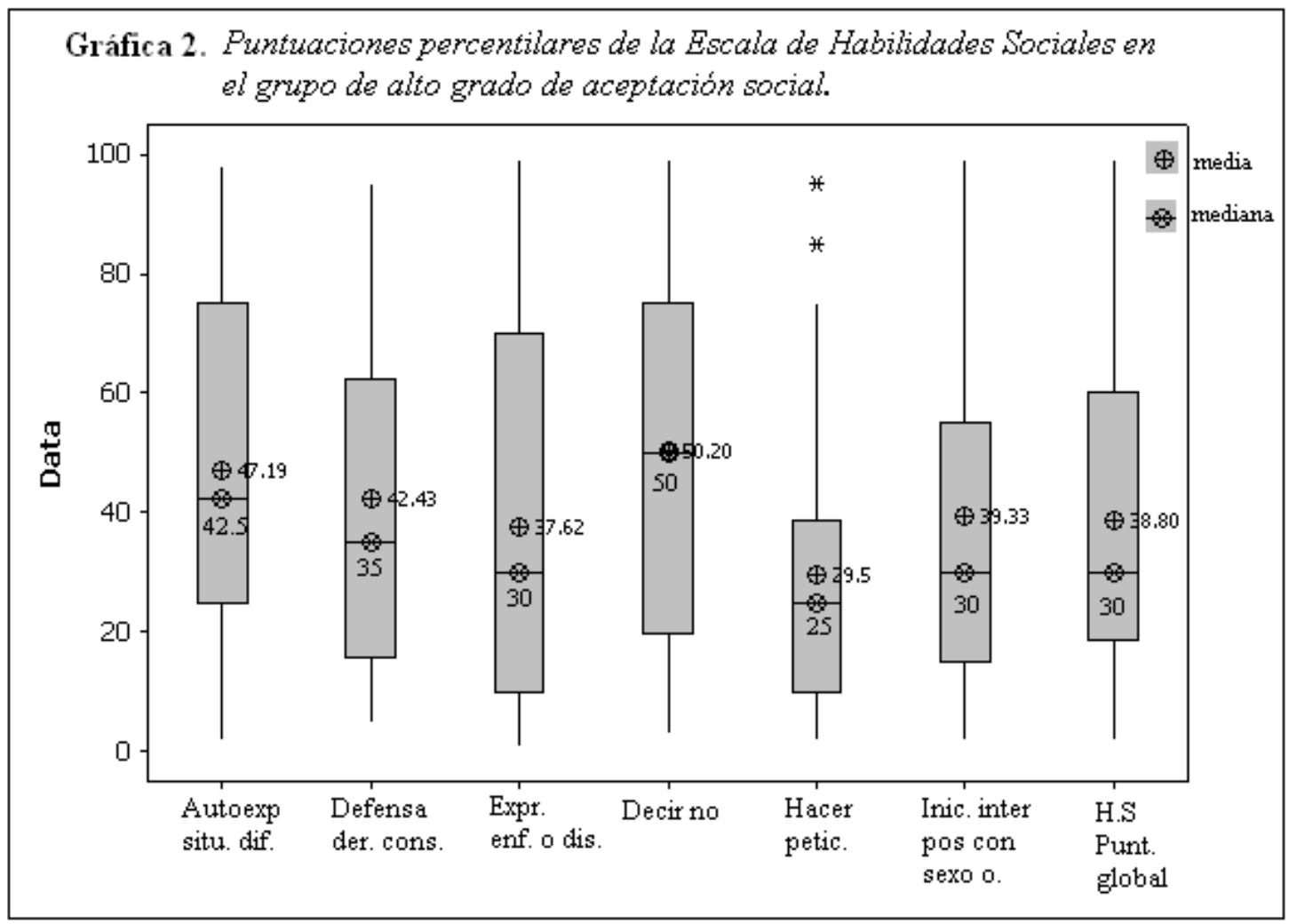

En el grupo con alta aceptación social, se efectuaron correlaciones de Spearman entre los factores del Bar-On y las variables de la Escala de Habilidades Sociales. Como puede observarse en la Tabla 2, se obtuvieron correlaciones positivas entre el factor Intrapersonal del Bar-On y la Habilidad para Decir No y Cortar Interacciones y la puntuación global de la Escala de Habilidades Sociales; el factor Interpersonal del Bar-On no obtuvo correlación con ninguno de los factores de la Escala de Habilidades Sociales. El factor Manejo del Estrés del Bar-On se correlacionó positivamente con el factor de Autoexpresión en Situaciones Difíciles. El factor de Adaptabilidad del Bar-On también se correlacionó positivamente con el factor de Autoexpresión en Situaciones Difíciles; además se correlacionó con los factores: Defensa de los Propios Derechos como Consumidor; Decir No y Cortar Interacciones; Iniciar Interacciones Positivas con el Sexo Opuesto; y con la puntuación global de Habilidades Sociales.

El Coeficiente Emocional del Bar-On se correlacionó positivamente con el factor de Autoexpresión en Situaciones Difíciles; Decir No y Cortar Interacciones; Iniciar Interacciones Positivas con el Sexo Opuesto; y con la puntuación global de Habilidades Sociales. El factor de Estado de Ánimo del Bar-On se correlacionó positivamente con el factor de Autoexpresión 
en Situaciones difíciles; Defensa de los Propios Derechos como Consumidor; Decir No y Cortar Interacciones; Iniciar Interacciones Positivas con el Sexo Opuesto; y, con la puntuación global de Habilidades Sociales. No se encontró ninguna correlación significativa entre los factores del Bar-On y los factores de Expresión de Enfado o Disconformidad y Hacer Peticiones de la Escala de Habilidades Sociales.

Tabla 2. Correlaciones entre los factores del Bar-On y los de la Escala de Habilidades Sociales en el grupo de alta aceptación social

\begin{tabular}{|c|c|c|c|c|c|c|c|}
\hline & $\begin{array}{c}\text { Autoex- } \\
\text { presión } \\
\text { en situa- } \\
\text { ciones } \\
\text { difíciles }\end{array}$ & $\begin{array}{c}\text { Defensa } \\
\text { de los } \\
\text { propios } \\
\text { derechos } \\
\text { como } \\
\text { consumi- } \\
\text { dor }\end{array}$ & $\begin{array}{c}\text { Expresión } \\
\text { de enfado } \\
\text { o discon- } \\
\text { formidad }\end{array}$ & $\begin{array}{c}\text { Decir no y } \\
\text { cortar } \\
\text { interac- } \\
\text { ciones }\end{array}$ & $\begin{array}{c}\text { Hacer } \\
\text { peticio- } \\
\text { nes }\end{array}$ & $\begin{array}{c}\text { Iniciar } \\
\text { interac- } \\
\text { ciones } \\
\text { positivas } \\
\text { con el } \\
\text { sexo } \\
\text { opuesto }\end{array}$ & $\begin{array}{c}\text { Habilida- } \\
\text { des } \\
\text { Sociales }\end{array}$ \\
\hline Intrapersonal & .239 & .240 & .157 & $.323^{* *}$ & .151 & .246 & $.366^{* *}$ \\
\hline Interpersonal & -.014 & .045 & -.028 & -.020 & -.061 & .048 & -.039 \\
\hline $\begin{array}{c}\text { Manejo del } \\
\text { estrés }\end{array}$ & $.292^{*}$ & .040 & .072 & .194 & -.051 & .166 & .208 \\
\hline $\begin{array}{c}\text { Adaptabili- } \\
\text { dad }\end{array}$ & $.390^{* *}$ & $.255^{*}$ & .224 & $.301^{*}$ & .029 & $.251^{*}$ & $.344^{* *}$ \\
\hline $\begin{array}{c}\text { Coeficiente } \\
\text { emocional }\end{array}$ & $.352^{* *}$ & .217 & .194 & $.313^{*}$ & .022 & $.273^{*}$ & $.335^{* *}$ \\
\hline $\begin{array}{c}\text { Estado de } \\
\text { ánimo }\end{array}$ & $.399^{* *}$ & $.281^{*}$ & .163 & $.405^{* *}$ & .165 & $.263^{*}$ & $.466^{* *}$ \\
\hline
\end{tabular}




\section{Discusión}

En el grupo de adolescentes con alta aceptación social se obtuvieron puntuaciones elevadas en todas las áreas de la IE auto-reportada, mismas que marcaron diferencias estadísticamente significativas con respecto del grupo natural, lo que es congruente con los hallazgos de la literatura que proponen una relación cercana entre inteligencia emocional y habilidad social, entre ellos se destacan los estudios de Lopes, Salovey y Straus (2003); Barchard y Hakstian (2004); Gil-Olarte, Palomera y Brackett (2006) y Mestre, Guil, Lopes y Salovey (2006), quienes señalaron que el reconocimiento y manejo de las emociones permean favorablemente las relaciones interpersonales y la adaptación social, especialmente entre los jóvenes.

En cuanto a las comparaciones por sexo es de llamar la atención que no hubo diferencias significativas en las puntuaciones de IE entre hombres y mujeres del grupo de alta aceptación social; mientras que en el grupo natural se encontraron diferencias significativas entre hombres y mujeres en algunos de sus componentes, de conformidad por los hallazgos reportados en varias investigaciones (Mayer y Geher, 1996; Mandell y Pherwani, 2003; Harrod y Scheer, 2005). Si se considera que tradicionalmente se motiva a las mujeres a jugar un rol más sensible a lo emocional a diferencia de los varones, se esperaría puntuaciones más elevadas en el caso de las mujeres, lo que se mostró parcialmente en el caso de las adolescentes del grupo natural; sin embargo, esto no sucedió en las adolescentes del grupo de alta aceptación social. Por otra parte, se observa que las mujeres de este grupo sí obtuvieron puntuaciones superiores con respecto de los varones del grupo natural a diferencia de sus compañeros varones con alta aceptación social; es comprensible que las puntuaciones de hombres y mujeres que se encuentran en los niveles superiores de IE, tiendan a aproximarse. Por otra parte, dado que las evidencias empíricas que relacionan IE y género arrojan resultados inconsistentes, es importante considerar una explicación plausible proporcionada por Fernández-Berrocal y Extremera (2006) quienes señalan que el tipo de instrumento utilizado es una variable a considerar ya que ya que en los cuestionarios y medidas de autoinforme como es el caso, no suelen encontrarse diferencias significativas, lo que sucede de manera contraria en las medidas de ejecución o desempeño como el MSCEIT en los que se han constatado claras diferencias significativas en IE a favor de las mujeres . 
Sobre la relación entre IE y Habilidades Sociales, a diferencia de los informes de Davies, Stankov y Roberts (1998), se encontraron correlaciones positivas entre la mayoría de los factores que las componen, confirmándose así los hallazgos de investigaciones empíricas que consideran que la IE es una variable clave en el mantenimiento de interacciones sociales positivas (Lopes, Salovey y Straus, 2003; Mestre, Guil, Lopes y Salovey, 2006; Weis y Süß, 2005). Con base en los resultados obtenidos podemos afirmar que los factores de la IE y las habilidades sociales están relacionados parcialmente; al respecto, se debe tener presente que ambos constructos fueron evaluados con instrumentos de auto-informe en los que se asume un posible sesgo por las creencias, la autopercepción y la deseabilidad social de los participantes.

Es importante emprender investigaciones, utilizando medidas objetivas de IE y desempeño social, que consideren los puntos de vista de otros actores sociales tales como padres, profesores y compañeros; así como estudios que relacionen la IE y las habilidades sociales mediante aproximaciones empíricas más finas que tomen en cuenta los ejes conductual, cognitivo, situacional y cultural en el que se manifiestan. Cuando se hace referencia a la IE y a las Habilidades Sociales, se debe tener en cuenta que no se trata de entidades conceptuales simples y homogéneas, es vital comprender su complejidad y precisar sus diferencias para facilitar el diseño de tratamientos y programas útiles y adecuados para los adolescentes.

\section{Agradecimientos}

Esta investigación fue financiada por el Consejo de Ciencia y Tecnología del Estado de Guanajuato (México), mediante el convenio 07-16-K662-075A01. 


\section{Referencias}

Bachard, K. A. y Hakstian, A. (2004). The nature and measurement of Emotional Intelligence abilities: Basic dimensions and their relations with other cognitive ability and personality variables. Educational and Psychological Measurement 64, (3), 437-462.

Bar-On, R. (1997). Bar-On Emotional Quotient Inventory: Technical manual.New York: Multi Health Systems

Bar-On, R. y Parker, J.D.A. (2004). Bar-On emotional quotient inventory: Youth version (Bar-On EQ-i:YV). Technical Manual. Toronto, Canadá: MHS.

Charbonneau, D. y Nicol, A. A. M. (2002). Emotional intelligence and prosocial behaviors in adolescents. Psychological Reports, 90 (2), 361-370.

Christoff, K. A., Scott, W.O. N., Kelley, M. L., Schlundt, D., Baer, G. y Kelly, J. A. (1985). Social skills, and social problem-solving training for shy young adolescents. Behavior Therapy, 16, 468-477.

Davies, M., Stankov; L., y Roberts, R. D. (1998). Emotional Intelligence: In search of an elusive construct. Journal of Personality and Social Psychology, 75, 989-1015.

Fernández-Berrocal, P. y Extremera, N. (2006). Emotional Intelligence: A theoretical and empirical review of its first 15 years of history. Psicothema, 18(supl.), 7-12.

Fernández-Berrocal, P., Extremera, N. y Palomera, R. (2008). Emotional intelligence as a crucial mental ability on educational context. In A. Valle, J. C. Núñez, R.G. Cabanach, J. A. González-Pineda and S. Rodríguez (Eds.). Handbook of Instructional Resources and Their Applications in the Classroom (pp. 67-88). New York: Nova Science Publishers, Inc.

Gil-Olarte, P., Palomera, R. y Brackett, M. A. (2006). Relating emotional intelligence to social competence and academic achievement in high school students. Psicothema, 18 (supl.), 118-123.

Gismero, E. (2002). Manual. Escala de Habilidades Sociales. (EHS). Madrid: TEA, Edicio nes, S. A.

Goleman, D. (2000). Inteligencia emocional.Buenos Aires: Javier Vergara.

Harrod, N. R. y Scheer, S. D. (2005). An exploration of adolescent emotional intelligence in relation to demographic characteristics. Adolescence (San Diego) an International Quarterly Devoted to the Physiological, Psychological, Psychiatric, Sociological and Educational Aspects of the Second Decade of Human Life, 40 (159), 503-524. 
Lopes, P. N., Salovey, P. y Straus, R. (2003). Emotional intelligence, personality and the perceived quality of social relationships. Personality and Individual Differences, 35(3), 641-659.

Mandell, B. y Pherwani, S. (2003). Relationship between emotional intelligence and transformational leadership style: a gender comparison. Journal of Bussiness and Psychoology, 17, 387-404.

Martínez-Pons, M. (1998). Parental inducement of emotional intelligence. Imagination, Cog nition and Personality, 18, 3-23.

Mayer, J. D. y Geher, G. (1996). Emotional intelligence and the identificación of emotion. Intelligence, 22, 433-442.

Mayer, J. D. y Salovey, P. (1997). What is emotional intelligence? En P. Salovey y D. Sluyter (Eds.). Emotional Development and Emotional Intelligence: Implications for Educators (pp. 3-31) New York: Basic Books.

Mayer, J. D., Salovey, P. y Caruso, D. R. (2000). Models of emotional intelligence. En R. J. Sternberg (Ed.). Handbook of Intelligence (pp. 396-420). Cambridge: Cambridge University Press.

Mestre, J. M., Guil, R., Lopes, P. N. y Salovey, P. (2006). Emotional intelligence and social and academic adaptation to school. Psicothema, 18, 112-117.

Parker, J. D. A., Creque, R. E., Barnhart, D. L., Harris, J. I., Majeski, S. A., Wood, L. M., Bond, B. J. y Hogan, M. J. (2004). Academic Achievement in high school: does emotional intelligence matter? Personality and Individual Differences 37, 1321-1330.

Petrides, K. V. y Furnham (2001). Trait emotional intelligence: Psychometric investigation with reference to established trait taxonomies. European Journal of Personality 15, 425-448.

Petrides, K.V., Frederickson, N. y Furnham, A. (2004). Personality and Individual Differences, 36, 277-293.

Thorndike, E. L. (1920). Intelligence and it uses. Harper's Magazine, 140, 227-235.

Trianes, M. V., De la Morena, M.L. y Muñoz, A .M. (1999). Relaciones sociales y preven ción de la inadaptación social y escolar. Málaga, España: Ediciones Aljibe.

Weis, S. y Süß, H. M. (2005). Social intelligence -A review and Critical Discussion of measurement concepts. In Ralf Schulze and Richar D. Roberts (Eds.). Emotional Intelligence. An Internacional Handbook (pp. 203-230). Göttingen, Germany: Hogrefe/Huber Publishers. 
Zirkel, S. (2000). Social intelligence. The development and maintenance of purposive behavior. En Reuven Bar-On y James D. A. Parker (Eds.). The Handbook of Emotional Intelligence. Theory, Development, Assessment, and Application at Home, School, and in the Work Place (pp. 3-27). San Francisco, CA EEUU: Jossey-Bas. 